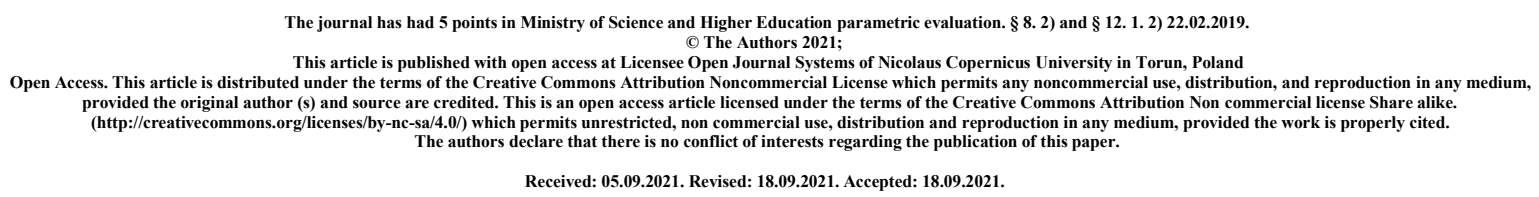

\title{
Xerostomia among elderly patients - systematic review
}

\author{
Martyna Drożak ${ }^{1}$, Paulina Drożak ${ }^{1}$, Justyna Dziekońska², Martyna Nowińska², \\ Paulina Grabowy ${ }^{2}$
}

1. Students' Scientific Association of Chair and Department of Family Medicine, Medical University of Lublin

2. Students' Research Group of Applied Psychology, Medical University of Lublin

\begin{abstract}
Introduction and purpose: Xerostomia is a salivary secretion malfunction that affects from 1 to $29 \%$ of the population. It is a common problem among elderly patients, however undeniably it still remains an underdiagnosed issue. Searching among available literature in the PubMed database with the following phrases: xerostomia; elderly, the aim of this article was to provide a broad review on the underdiagnosed problem which is xerostomia among elderly patients.

Description: A group at risk of developing xerostomia are people over 65 years old and women in the perimenopausal period. Although dry mouth varies in etiology, geriatric patients mostly develop xerostomia as a result of head and neck radiotherapy, Sjögren syndrome or medication treatment. Untreated symptoms can lead to severe issues which heavily impact not only oral health of the patient, but also their everyday life quality, since xerostomia may lead into difficulty of speaking, swallowing and tasting. The plan of treatment is influenced by the etiology of the case, however it is aimed to stimulate salivary
\end{abstract}


flow and eradicate the use of unnecessary medication which may cause dry mouth. Prevention of dry mouth is based on maintenance of good oral hygiene.

Conclusions: Diagnosing the problem early can prevent patients from suffering the consequences of chronic xerostomia, therefore awareness should be brought to this issue. Dentist could also highly improve the quality of xerostomic patient's life, if the chosen treatment significantly improved patient's symptoms.

Key words: xerostomia; elderly

\section{Introduction}

The daily secretion of saliva ranges from $500 \mathrm{ml}$ to $700 \mathrm{ml}$, with an average saliva flow of 0,3 $-0,4 \mathrm{ml} / \mathrm{min}[1,2] .93 \%$ of saliva is produced by the major salivary glands present in the oral cavity - sublingual glands, parotid glands and submandibular glands $[1,3]$. The other $7 \%$ is secreted by minor glands, located in every region of oral cavity apart from the anterior section of the hard palate and the gums [1].

Saliva has a very complex role in our organisms [4]. It takes a major part in digestive function, swallowing, tasting, chewing, speaking, phonation and remineralization process of the teeth [5]. It also plays a crucial role in lubricating surfaces present in oral cavity and maintaining homeostasis inside the mouth. The composition of saliva differs between individuals and is heavily dependent on the time of the day. Protein buffer, phosphate and bicarbonate are responsible for the buffer capacity of saliva. Saliva also contains immunoglobulins, which successfully neutralize toxins or viruses entering the oral cavity. Inorganic components such as bicarbonate, phosphate and calcium play a crucial role in prevention of demineralization of the teeth and intensify the remineralization of enamel. An enzyme called amylase is responsible for the first stage of carbohydrates digestion, whereas lipase initiates fat digestion [2]. The $\mathrm{pH}$ of saliva ranges from 6,8 to 7,2 and is maintained by phosphate buffering system and hydrogen carbonate, which prevents acids produced by bacteria from damaging oral cavity [6,2]. Therefore, any failure of salivary function may lead to extensive side effects [5]. 
Malfunction associated with salivary secretion are commonly divided into three groups: hyposalivation, xerostomia and changes in the composition of saliva [5]. „Dry mouth” is a term used to describe both xerostomia and hyposalivation [5]. However, xerostomia is defined as a subjective feeling of dehydration of oral cavity caused by insufficient or no saliva secretion $[7,6]$. It is a problem that affects $1-29 \%$ of the population. Groups most likely to be affected by xerostomia are women in the perimenopausal period and people over 65 years of age [6]. Hyposalivation is described as major reduction of salivary flow [5]. Xerostomia is often associated with hyposalation, but there are cases of xerostomia in which the rate of salivation is normal. Hyposalation is the phenomenon of saliva secretion at a rate below 0.1 $\mathrm{ml} / \mathrm{min}$ at rest and $0.7 \mathrm{ml} / \mathrm{min}$ during stimulation [8].

Xerostomia is classified based on patogenesis of the issue as "true xerostomia" and "pseudo xersotomia". In true xerostomia, dry mouth is caused by abnormal functioning of the salivary glands. On the other hand, in the case of pseudo-xerostomia, dry mouth is only a subjective feeling when the salivary glands function within the normal range [6].

\section{Etiology of xerostomia}

Xerostomia should be looked after radiotherapy of the head and neck, some systemic diseases and the use of certain medications [8]. Systemic diseases that may cause symptoms pf xerostomia include neurological diseases (depression, Parkinson's disease), metabolic diseases (bulimia, dehydration, anemia, alcohol addiction), genetic diseases, endocrine disorders (hypothyroidism, hyperthyroidism and DM), inflammatory diseases and rheumatology (Sjögren's syndrome, systemic lupus erythematosus, rheumatoid arthritis), viral diseases (HCV, AIDS) and other [8]. Some cases of dry mouth have psychiatric origins and occur together with diseases such as depression or neurosis [8]. Although in these cases, xerostomia may also originate as a side effect of taking tryciclic antidepressants, antipsychotic or anticonvulsant medication and sedative [9].

The most common cause of xerostomia among geriatric patients is medication intake, because, according to studies, seniors take at least one drug that negatively affects the work of the salivary glands [10]. According to literature, there is about 500 drugs significantly inducing xerostomia [11]. Interactions between certain types of medication might also cause a synergystic effect which will heavily impact the function of salivary gland $[11,6]$. 
Xerostomia- inducing medications include drugs for epilepsy, obesity, diarrhea, asthma (some bronchodilators), urinary incontinence, Parkinson's disease and hypertension [12]. Drugs with potential xerostomic effects that are most frequently used by elderly patients include opiates, muscle relaxants, anticholinergic, antihistamines, sedative-hypnotics and sympathomimetic [13].

Radiotherapy of head and neck is also listed as one of the common causes of xerostomia. Apart from dry mouth, exposure to radiation can lead to parenchymal loss and sialodentitis. The extent of impact of radiation is heavily dependent on the dose of radiation, the area exposed to radiant energy, the time of exposure, the type of radiation etc. However, it was proven that the amount of both resting and stimulated saliva heavily decreased after the exposure to radiation [9].

Studies have shown that in approximately $40 \%$ of xerostomia cases among elderly population, Sjögren syndrome is a factor which causes symptoms of dry mouth [14]. This autoimmune disease is a malfunction of exocrine glands which leads not only to xerostomia, but also other severe manifestations in oral cavity or eyes, such as keratitis sicca [15]. It can also affect gastrointestinal system, respiratory system, skin, vagina, liver, joints, kidneys or vascular, musculoskeletal and central nervous system. However, Sjögren syndrome is said to be one of the most underdiagnosed illness, since the characteristics of this condition are often wrongly mistaken for features of physiological aging or the after effects of pharmacological treatment [14].

Xerostomia as a result of aging still remains a controversial subject [9]. Clinical studies have shown that in most cases of geriatric patients the salivary glands do not show signs of malfunction. Therefore, xerostomia is most likely not a result of physiological aging, but induced by an external factor. However, in a view of available data, saliva significantly changes its chemical composition with age. The concentration of mucins increases, while the concentration of pythialin decreases, making the saliva thicker and more viscous which can cause health problems in the population of seniors [16].

Certain lifestyle choices can also affect the salivary flow. Chewing tobacco or smoking can lead to the development of dry mouth symptoms [12]. Smoking is also heavily linked to other dental disorders related to xerostomia, such as higher risk of developing dental calculus, cervical caries, tooth mobility, halitosis and gingivitis [17]. Patients who frequently wear 
removable dentures are also five times more likely to start having dry mouth than patients without the dentures [9].

\section{Symptoms and complications}

The most common symptom associated with xerostomia is discomfort due to dryness of the mouth [6]. Decreased salivary flow and overall smaller volume of saliva in oral cavity can negatively affect immunological and non-immunological barrier which often leads to farreaching consequences, such as problems with eating solid foods, taste disturbance, BMS (burning mouth syndrome), fetor ex ore, ruptures of soft tissues surrounding the oral cavity (especially lips), „milky” saliva near the area of salivary duct openings [6]. Patients suffering from Sjögren's syndrome also report sensation of excessive dryness in the eyes, nose, lips and throat area [9].

Severe xerostomia often leads to developing complications, for example increased risk of buccodental disease (gingivitis, erosion, dental caries, ulceration of mucosal tissues, oral candidiasis, periodontal disease, angular cheilitis and mucositis). Cervical caries are often developed by the patients due to accumulation of excessive amounts of dental plaque at surfaces of the tooth which are normally not predisposed to coaggregate plaque. These surfaces include cusps of lateral teeth or the front of mandibular teeth. Patients mostly develop Black class V caries [6]. Speech impairment is stated as a consequence of malfunction in salivary secretion [5]. Dysgeusia and dysphagia are also far-reaching consequences of xerostomia, mostly reported by geriatric patients $[9,18,5]$. Elderly patients suffer from increased tooth sensitivity or monilial infections as well. Adequate oral hygiene is indeed a crucial factor in prevention of the development of xerostomia [18].

A diagnosis of dry mouth is mainly based on various methods which compare salivary flow rate during stimulation and at rest to standard measurements [19]. Among those methods, sialometry is the most widely used one. It is a procedure which collects whole saliva or a part of stimulated or unstimulated saliva secreted by each major salivary gland [5]. 


\section{Treatment}

In the available literature, no clear standards for the treatment of xerostomia were wellestablished. The treatment of dry mouth is chosen based on etiology of the case, systemic treatments received by the patient, prevention and local salivary stimulation [12]. It mostly focuses on selecting effective medications for underlying disease and refraining from any unessential medicine [19].

When it comes to Sjögren's syndrome, the therapy mostly focuses on treating the symptoms and may need systemic immunosuppressive and immunomodulatory agents as a part of pharmacological treatment to control other manifestations of the condition [20,21]. Studies have shown that the most effective drug treatment, which shows major improvements in salivary secretion and reduction of symptoms, is pilocarpine. Pharmacological treatment which includes usage of malic acid and rebamipide was also investigated, but failed to prove its efficiency due to no significant differences between the tested group and placebo [13]. Oral dryness in the vast majority of cases is kept under control also by maintaining good oral hydration and refraining from medication increasing oral dryness. Patients should be informed about possible risk factors that may intensify condition, for example breathing through the mouth. It is also advised for the patient to regularly drink oral solutions and suck sugar-free candy in order to stimulate the saliva flow. Keeping a good oral hygiene is also crucial to prevent other dental problems which are caused by dryness of the mouth [21].

Patients whose xerostomia was caused by radiotherapy of head and neck may also require parasympathomimetic drugs as a form of pharmacological treatment [19]. The most common drug to use in this case is pilocarpine [13]. Medication such as cevimeline and bethanechol have also shown positive results in improving salivary secretion. However, it remains unknown whether usage of those drugs is more effective and beneficial to the patient than treatment with pilocarpine [13].

\section{Summary}

In conclusion, xerostomia is a severely underdiagnosed disease that heavily impacts wellbeing of the patients. Since life expectancy in the past years has significantly changed, such inconveniences as salivary problems associated with xerostomia require special attention from 
the dentists' side. Despite the fact that no clear link between ageing and malfunction of salivary secretion was established, it is a common problem among elderly patients. It mostly originates as a result of certain medication intake, however such cases have shown major improvements after reduction of the xerostomia-inducing drug. Cases with different etiologies of the disease vary in treatment, however, early prevention of this disease can significantly improve oral health among geriatric population and lessen the risk of developing salivary secretion problems. The following article provided a broad review of the matter which is xerostomia among elderly.

\section{References:}

[1] Llena-Puy C. The rôle of saliva in maintaining oral health and as an aid to diagnosis. Med Oral Patol Oral Cir Bucal. 2006 Aug;11(5):E449-55. English, Spanish. PMID: 16878065.

[2] Kaczor-Urbanowicz KE, Wei F, Rao SL, Kim J, Shin H, Cheng J, Tu M, Wong DTW, Kim Y. Clinical validity of saliva and novel technology for cancer detection. Biochim Biophys Acta Rev Cancer. 2019 Aug;1872(1):49-59. doi: 10.1016/j.bbcan.2019.05.007. Epub 2019 May 30. PMID: 31152821; PMCID: PMC6692231.

[3] Zhang CZ, Cheng XQ, Li JY, Zhang P, Yi P, Xu X, Zhou XD. Saliva in the diagnosis of diseases. Int J Oral Sci. 2016 Sep 29;8(3):133-7. doi: 10.1038/ijos.2016.38. PMID: 27585820; PMCID: PMC5113094.

[4] Carpenter GH. The secretion, components, and properties of saliva. Annu Rev Food Sci Technol. 2013;4:267-76. doi: 10.1146/annurev-food-030212-182700. PMID: 23464573.

[5] Islas-Granillo H, Borges-Yáñez A, Fernández-Barrera MÁ, Ávila-Burgos L, Patiño-Marín $\mathrm{N}$, Márquez-Corona ML, Mendoza-Rodríguez M, Medina-Solís CE. Relationship of hyposalivation and xerostomia in Mexican elderly with socioeconomic, sociodemographic and dental factors. Sci Rep. 2017 Jan 17;7:40686. doi: 10.1038/srep40686. PMID: 28094800; PMCID: PMC5240562.

[6] Tanasiewicz M, Hildebrandt T, Obersztyn I. Xerostomia of Various Etiologies: A Review of the Literature. Adv Clin Exp Med. 2016 Jan-Feb;25(1):199-206. doi: 10.17219/acem/29375. PMID: 26935515. 
[7] Bivona PL. Xerostomia. A common problem among the elderly. N Y State Dent J. 1998 Jun-Jul;64(6):46-52. PMID: 9707984.

[8] López-Pintor RM, Casañas E, González-Serrano J, Serrano J, Ramírez L, de Arriba L, Hernández G. Xerostomia, Hyposalivation, and Salivary Flow in Diabetes Patients. J Diabetes Res. 2016;2016:4372852. doi: 10.1155/2016/4372852. Epub 2016 Jul 10. PMID: 27478847; PMCID: PMC4958434.

[9] Villa A, Abati S. Risk factors and symptoms associated with xerostomia: a cross-sectional study. Aust Dent J. 2011 Sep;56(3):290-5. doi: 10.1111/j.1834-7819.2011.01347.x. PMID: 21884145 .

[10] Gupta A, Epstein JB, Sroussi H. Hyposalivation in elderly patients. J Can Dent Assoc. 2006 Nov;72(9):841-6. PMID: 17109806.

[11] Shetty SR, Bhowmick S, Castelino R, Babu S. Drug induced xerostomia in elderly individuals: An institutional study. Contemp Clin Dent. 2012 Apr;3(2):173-5. doi: 10.4103/0976-237X.96821. PMID: 22919217; PMCID: PMC3425100.

[12] Alsakran Altamimi M. Update knowledge of dry mouth- A guideline for dentists. Afr Health Sci. 2014 Sep;14(3):736-42. doi: 10.4314/ahs.v14i3.33. PMID: 25352896; PMCID: PMC4209628.

[13] Gil-Montoya JA, Silvestre FJ, Barrios R, Silvestre-Rangil J. Treatment of xerostomia and hyposalivation in the elderly: A systematic review. Med Oral Patol Oral Cir Bucal. 2016 May 1;21(3):e355-66. doi: 10.4317/medoral.20969. PMID: 27031061; PMCID: PMC4867210.

[14] Al-Hashimi I. Xerostomia secondary to Sjögren's syndrome in the elderly: recognition and management. Drugs Aging. 2005;22(11):887-99. doi: 10.2165/00002512-20052211000001. PMID: 16323968.

[15] Cartee DL, Maker S, Dalonges D, Manski MC. Sjögren's Syndrome: Oral Manifestations and Treatment, a Dental Perspective. J Dent Hyg. 2015 Dec;89(6):365-71. PMID: 26684993.

[16] Astor FC, Hanft KL, Ciocon JO. Xerostomia: a prevalent condition in the elderly. Ear Nose Throat J. 1999 Jul;78(7):476-9. PMID: 10429321.

[17] Rad M, Kakoie S, Niliye Brojeni F, Pourdamghan N. Effect of Long-term Smoking on Whole-mouth Salivary Flow Rate and Oral Health. J Dent Res Dent Clin Dent Prospects. 
2010 Fall;4(4):110-4. doi: 10.5681/joddd.2010.028. Epub 2010 Dec 21. PMID: 23346336; PMCID: PMC3429961.

[18] Navazesh M. Xerostomia in the aged. Dent Clin North Am. 1989 Jan;33(1):75-80. PMID: 2642824.

[19] Närhi TO, Meurman JH, Ainamo A. Xerostomia and hyposalivation: causes, consequences and treatment in the elderly. Drugs Aging. 1999 Aug;15(2):103-16. doi: 10.2165/00002512-199915020-00004. PMID: 10495070.

[20] Witte T. Sjögren-Syndrom [Sjögren's syndrome]. Z Rheumatol. 2014 Feb;73(1):49-61. German. doi: 10.1007/s00393-013-1313-8. PMID: 24554256.

[21] Baer AN, Walitt B. Update on Sjögren Syndrome and Other Causes of Sicca in Older Adults. Rheum Dis Clin North Am. 2018 Aug;44(3):419-436. doi: 10.1016/j.rdc.2018.03.002. PMID: 30001784; PMCID: PMC6245643. 